\title{
Child and adolescent psychiatric disorders in a public service over seventy years
}

\author{
Steinhausen, Hans Christoph
}

\begin{abstract}
The entire sample of $\mathrm{N}=45554$ patients attending the child and adolescent psychiatric service of the Canton of Zurich, Switzerland between 1921 and 1990 was analyzed with regard to age, sex, and diagnoses. Data were based on annual reports of the institution for the period between 1921 and 1978 . From 1979 onwards, electronically stored data on each patient were available. ICD-9 diagnoses were applied between 1979 and 1987. The ICD-10 system was introduced in 1988. The total administrative prevalence rate varied between 0.15 and 0.40 percent with an increasing trend over time. On the average, boys outnumbered girls by a sex ratio of $2: 1$. The age distribution showed peaks at 8 to 9 years and during adolescence. The distributions of the most common diagnoses showed remarkable differences over time. It is assumed that changes in diagnostic categories rather than changes in true prevalence rates play a major role in the latter trend
\end{abstract}

DOI: https://doi.org/10.1007/bf00573639

Posted at the Zurich Open Repository and Archive, University of Zurich ZORA URL: https://doi.org/10.5167/uzh-156334

Journal Article

Published Version

Originally published at:

Steinhausen, Hans Christoph (1997). Child and adolescent psychiatric disorders in a public service over seventy years. European Child Adolescent Psychiatry, 6(1):42-48.

DOI: https://doi.org/10.1007/bf00573639 
H.-Ch. Steinhausen

Received: 8 December 1995

Accepted: 20 February 1996

Prof. Dr. Dr. H.-Ch. Steinhausen (

Department of Child

and Adolescent Psychiatry

University of Zürich

Freiestraße 15

8028 Zürich, Switzerland

\section{Child and adolescent psychiatric disorders in a public service over seventy years}

trend over time. On the average, boys outnumbered girls by a sex ratio of 2:1. The age distribution showed peaks at 8 to 9 years and during adolescence. The distributions of the most common diagnoses showed remarkable differences over time. It is assumed that changes in diagnostic categories rather than changes in true prevalence rates play a major role in the latter trend.

Key words Child psychiatric disorders - epidemiology time trends

\section{Introduction}

One of the questions repeatedly posed to academic child and adolescent psychiatry concerns whether or not mental disorders in children and adolescents have become more common in the recent past and the present. A related question deals with the issue of structural change, i.e., whether or not the distribution of disorders has changed in terms of some diagnoses becoming less or more frequent over time. An attempt to analyze these questions is fraught with two major problems. The first is an epidemiological problem, i.e., the rarity of an adequate data base, whereas the second refers to a nosological problem, i.e., a system of classification of diagnoses that is stable over time.

Ideally, the epidemiological problem could only be solved by having access to a series of representative cross-sectional studies in which data on mental health of children and adolescents is repeatedly collected over time in a given region. Despite the enormous increase of more and more sophisticated epidemiological studies in the field of child and adolescent psychiatry $(4,9,13$, $14,18)$ no series of studies of this type exists. Thus, at present, there is no data base available to compare true prevalence rates of mental disorders in children and adolescents over time.

Given this restriction, one is referred to psychiatric registers in which data on the morbidity of a population is accumulated over a sufficient span of time in order to analyze temporal changes. Regrettably, studies based on psychiatric registers are fraught with another major research restriction insofar as no true prevalence rates but rather only administrative prevalence rates can be obtained. However, there are only very few countries in the world, most notably Scandinavian countries, in which there is a long-standing tradition of these kinds of registers, and where only recently public objection against using them for research purposes has developed. Accordingly, these registers have been used for followup studies on the long-term outcome of former child 
and adolescent psychiatric patients $(12,16)$ or for family studies on the transmission of psychiatric disorders such as schizophrenia, depression, and alcoholism. Interestingly, so far, these Scandinavian psychiatric registers have not been used for the analysis of temporal changes in child and adolescent morbidity.

When restricting the analysis of the frequency and temporal changes in child and adolescent psychiatric morbidity to register data, one is confronted with an additional nosological problem. Both in psychiatry and in child and adolescent psychiatry, there was a long history of various schools of thought in many regions of the world leading to a multitude of different nosological schemes. This led to major problems in communication across countries and schools of thought. In psychiatry, as in other fields of medicine, two lines of development helped to overcome these major obstacles to research, namely the design of international classifications of diseases (ICD) and, concurrently, the major technological advances in the field of data computation that came up in the recent decades.

With the development of the ICD-9 (26) and its successor, the ICD-10 (27), and in parallel, the various forms of the Diagnostic and Statistical Manual of Mental Disorders (DSM) $(1,2,3)$ researchers are in a much better situation since the 1970's and 1980's to analyze epidemiological trends across regions and time. However, a remaining problem is the limited comparability of the diagnostic categories based on either the more recent classification systems or the more traditional psychiatric categories. In some instances, there is a greater continuity than in others. These changes in the definition of certain psychiatric disorders may impose further limitations on the analysis of temporal changes in child and adolescent psychiatric morbidity.

Accordingly, the present study is also fraught with various methodological problems. However, it was undertaken to shed some light on the interesting question of morbidity changes across time. It is based on a large case register for patients who attended the Child and Adolescent Psychiatric Service of the Canton of Zurich, Switzerland between 1921 and 1990. Since it was founded in 1921, the service was always strongly community based. From 1921 to 1931, it consisted only of a small in-patient unit. After this first decade, a first out-patient clinic was opened. Shortly after the war, in 1947, the second out-patient clinic was added to the service. In the middle of the seventies, the continuation of this development had led to the installation of a total of seven out-patient clinics across the entire canton, a major in-patient unit for children, and a day clinic for children. With the exception of a lack of in-patient treatment facilities for adolescents, this service represents one of the most differentiated and largest community-based child and adolescent services in Europe.
The patients attending this service were representative of a long period insofar as the first five child and adolescent psychiatrists began to establish private practices only from 1955 onwards. Their numbers increased constantly from that time onwards. In 1990, there were a total of 34 child and adolescent psychiatrists working in private practice in the canton. At this time, 60 clinicians were working in the child and adolescent psychiatric service including senior and junior child psychiatrists and psychologists.

\section{Materials and methods}

\section{Subjects}

Data of the present study were obtained from a total sample of $\mathrm{N}=45554$ patients, aged $0-19$ years, who attended the Child and Adolescent Psychiatric Service of the Canton of Zurich, Switzerland between 1921, the year that the service was founded, and 1990. Accordingly, a time span of seventy years is covered. The sex and age distribution will be shown with the findings. There were no socioeconomic status data available across the entire time span. Currently, in the early 1990 's, approximately fifty percent are of lower class origin, forty percent of middle class origin, and ten percent come from the upper classes.

According to the official census, the population of the Canton of Zurich that was between the ages of zero and nineteen years of age increased from 152884 in 1920 to 238709 with a peak of 301972 in 1970 . The canton comprises both urban and rural areas.

\section{Procedure}

From its beginning, the history of the service was documented with annual reports that were obligatory and had to be given to the political administration. These annual reports include summary statistics of the clientele of children and adolescents who attended the service for the first time in each respective year. These sources were used in the present study for the time span between 1921 and 1978. Since 1979, a data bank is available that includes personal, sociodemographic, and diagnostic data. The ICD-9 system was used from 1979 to 1987 and was followed by the ICD-10 scheme. Each patient was classified only with one diagnosis throughout the period from 1921 to 1987. Multiple diagnoses have only been possible since 1988 , i.e., for the last three years of data collection. In these instances, only the first diagnosis was considered in the analyses. 
Fig. 1 Attendance

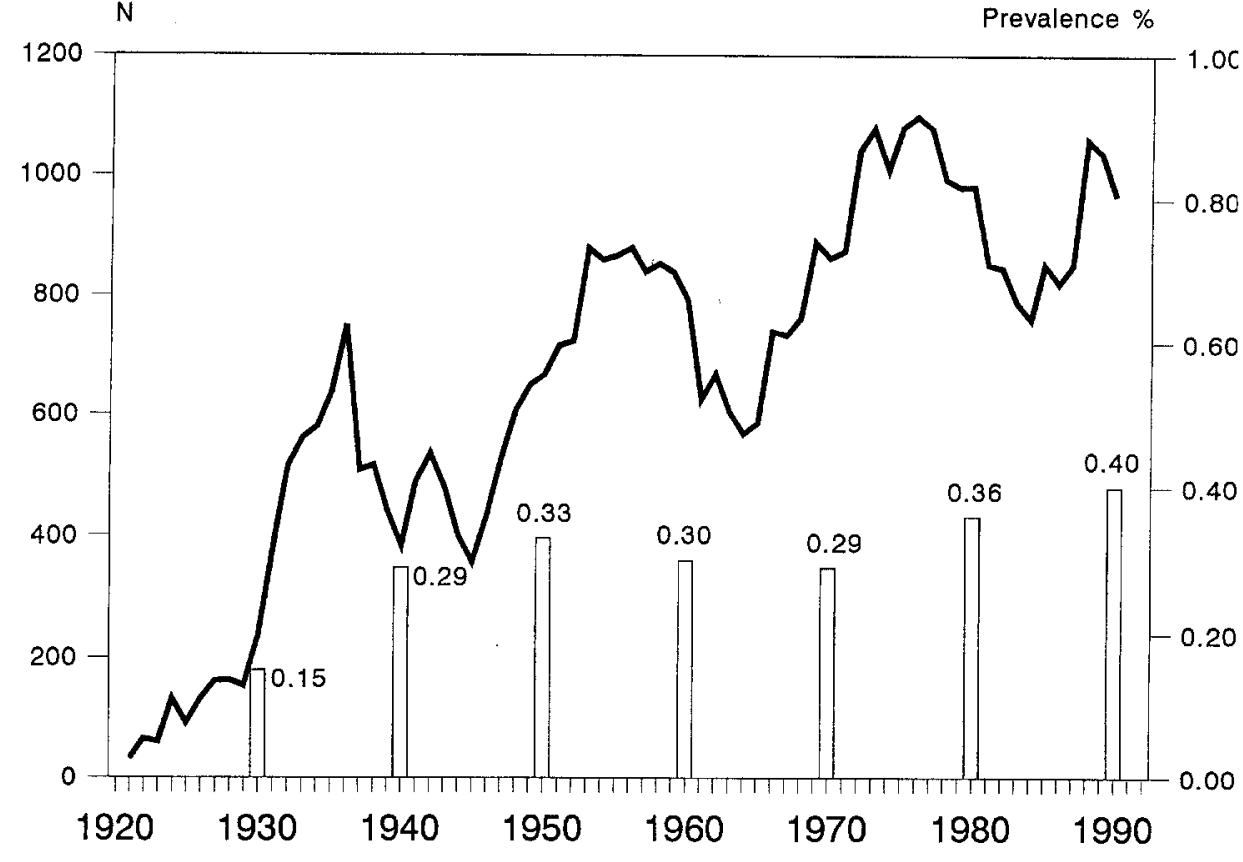

Fig. 2 Sex distribution



The structure of the data bank has been subjected to various revisions and formed the basis for various recent studies on classification issues (22-25). The following data on prevalence rates, age and sex distribution, and diagnoses across time are shown in a descriptive way. No statistical tests were carried out because, in a sample of this size, even minor changes would turn out to be significant.

\section{Results}

The number of children and adolescent attending the service did not develop steadily but rather in a sine curve fashion with a tendency to increase over time, as shown in Fig. 1. There is a similar tendency in the administrative prevalence rates. However, the two curves do not run parallel and it has to be taken into account that the absolute frequencies are based on annual statistics, whereas the prevalence rates could be obtained 
Fig. 3 Age distribution
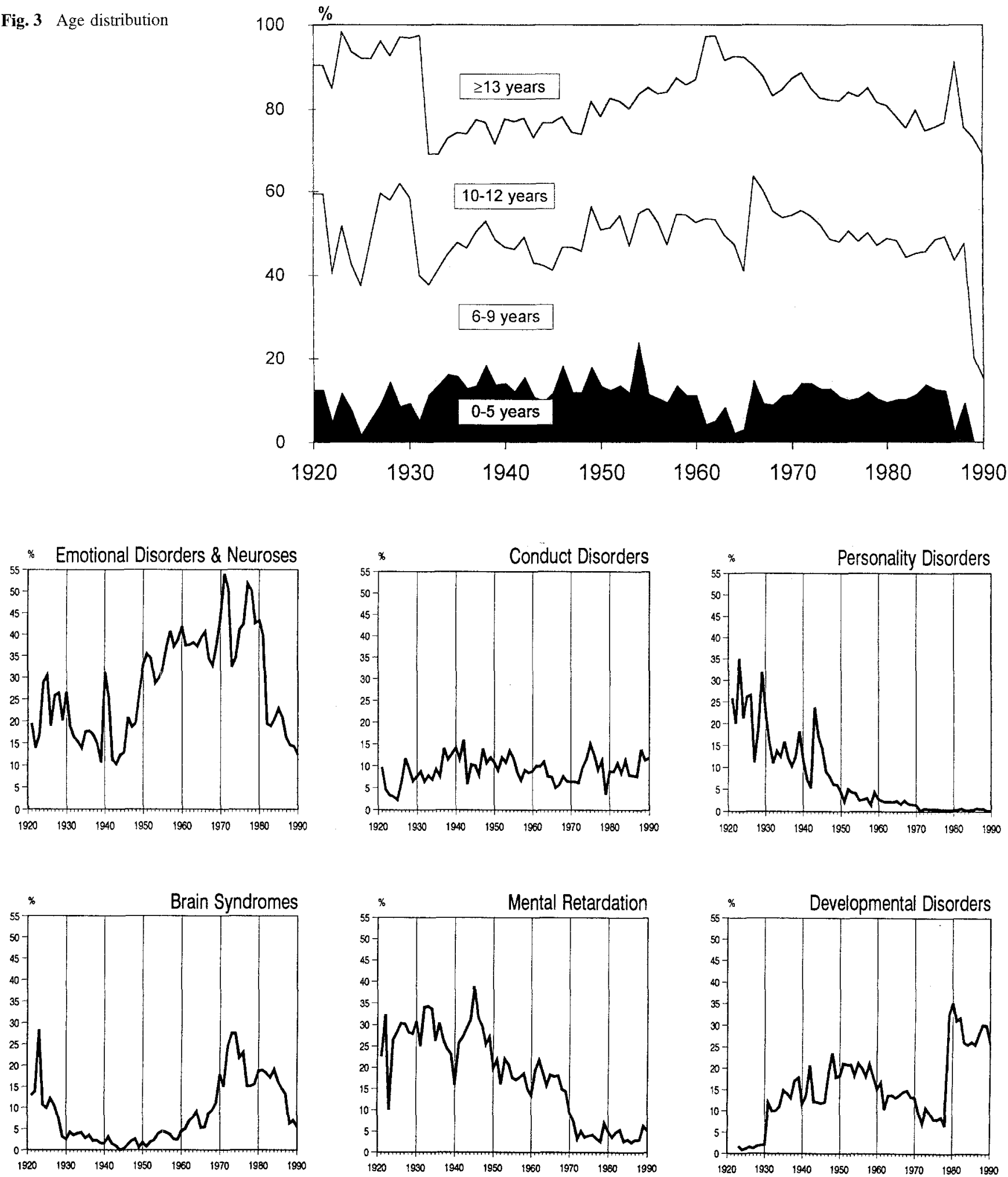

Fig. 4 Distribution of diagnoses 
only at seven points of time - at the beginning of a new decade when official census data were available. The administrative prevalence rates range between 0.15 percent in 1930 and 0.40 percent in 1990.

The sex disribution, as given in Fig. 2, shows a clear preponderance of boys across time. With the exception of the early years in the 1920's, when the absolute figures were relatively small, boys outnumber girls with an average of $2: 1$ sex ratio. After the first decade, with very few adolescents attending the service, the age distribution across the remaining period of the further sixty years shows a predominance of children aged six to nine and ten two twelve years. In the early 1960s there was, again, a decrease of adolescents and, in addition, of very young children attending the service (see Fig. 3).

The distribution of diagnoses was studied only for the most common categories in which some continuity in the definition across time could also be assumed. The findings are displayed in Fig. 4. There are some remarkable changes for various distributions across time. It becomes apparent that emotional and neurotic disorders were most frequently diagnosed in the period from the 1950's onwards with peaks in the 1970's. With the introduction of the ICD-9 and the ICD-10 schemes, the frequencies dropped considerably. In contrast, there is very little variation for conduct disorders across time. Personality disorders were more commonly diagnosed in the period before and during the Second World War whereas, thereafter, the frequencies are very low.

For brain syndromes there is a first peak in the early 1920 's, which is negligible because of the very small absolute figures of the clientele at that time. More importantly, there is an interesting peak in the 1970's with the curve tapering off from that time and rather low frequencies in the late 1980's concurrent to the introduction of the ICD-10. The curve for mental retardation shows some variation on a rather high level between the 1920's and the 1940's and then declines continuously from the 1950s onwards. Finally, the distribution for developmental disorders shows some fluctuation between approximately 10 to 20 percent for most of the time span and a striking increase from 1979, with the introduction of the ICD-9 scheme.

\section{Discussion}

This study provides data on attendance rates, administrative prevalence rates, sex and age distributions, and diagnoses across a time span of seven decades. The attendance rates showed a sine-like curve distribution with increasing frequencies across time. One may assume that various factors may account for this phenomenon, i.e., true morbidity changes, availability of the service, information about the service, and historical or societal trends at large. Whereas no data are available for the true morbidity changes per se, there is some evidence that the availability of the service and, concomitantly, the information about the service did contribute to attendance rates. Clearly, the extension of the service with the opening of additional out-patients clinics in 1931, 1947, 1948, and 1970 coincided with an increase in attendance rates. However, the same event in 1953 and 1975 did not have a similar effect.

Whether the administrative prevalence figures follow a similar pattern of a sine-like curve with an increasing tendency is not absolutely certain because of the smaller data base with seven instead of seventy measurements. However, the increasing tend over time of $0.15 \%$ in 1930 to $0.40 \%$ in 1990 is noteworthy. This trend could imply that with increasing information and availability of treatment facilities, a larger proportion of the true morbidity was detected. One need not necessarily conclude that this trend is also an indication of an increasing morbidity. This conclusion might even hold true if one takes into account the fact that, besides the child and adolescent psychiatric service, further sources of treatment such as child and adolescent psychiatrist in private practice, school psychological services, or child guidance clinics extended the existing treatment facilities. It simply could mean that over time, an increasing proportion of the true prevalence was identified in the service.

Without knowing the exact true prevalence, one has to assume that a rather wide gap exists between administrative prevalence and true prevalence. This conclusion is warranted on the grounds of large epidemiological surveys in which median prevalence rates were obtained for child and adolescent psychiatric disorders of 11.8 percent for the USA and 13.2 percent for Great Britain (9) or 14-20 percent in recent surveys in various Anglo Saxon and European countries (4). Additional evidence for this conclusion comes from several epidemiological studies that show that only a small proportion of the true prevalence is identical with the administrative prevalence, indicating that only a minority of identified cases do actually receive treatment $(7,15,19)$.

Both the sex and age distributions observed in this study are in accordance with epidemiological studies. The present 2:1 sex ratio in males has also been obtained in various field studies (21) underscoring the greater psychiatric vulnerability of boys. Similarly, the high proportion of psychiatric disorders in late childhood and in adolescence has been notified (14).

Comparability of diagnoses across time was sought by restricting the diagnoses to a few broad categories. Because of relying on single diagnoses until 1987 and allowing for multiple diagnoses only since 1988, there should be no noticeable methodological bias in the data set. The distribution of diagnoses revealed some strik- 
ing changes over time. These variations may to a greater extent reflect changes in the child psychiatric prevailing schools of thought than in true prevalence. For instance, the increase in frequencies of emotional and neurotic disorders in the period from the 1950's to the 1970's coincided with the influence of the psychoanalytic theory that was dominant both in Switzerland and in other countries as well as with the transformation of the local psychotherapeutic service towards an increase of psychotherapeutic facilities in contrast to the traditional approach that was more educational and custodial. During this period, diagnostic terms were used rather vaguely, and this applied especially to the diagnostic category of so-called 'neurotic reactions'. This was brought to an end with the upcoming and more precise definitions of the ICD-9 scheme and, even more definitively, with the more strict criteria and diagnostic guidelines of the ICD-10.

In contrast, no such effects could be observed for conduct disorders, for which the distribution remained rather stable over seventy years. Obviously, this category has always been defined on phenomenological and behavioural grounds and was less open to definitions based on theoretical constructs such as the term 'neurosis'.

Interestingly, in the present study, there was a remarkable decrease in the distribution of personality disorders across time with an almost total disappearance in the recent past. This trend certainly reflects the increasing reluctance of many child and adolescent psychiatrists to use a diagnostic category of fixed outcome that runs counter to their basic assumptions of an open and fluid development (10). Again, rather than any changes in true prevalence rates, the most plausible explanation for these apparent trends is a change in the child and adolescent psychiatric culture from a more static theory of psychopathology to a more dynamic conceptualization of mental disorders in children and adolescents.

Similar trends may also be assumed for the changes of frequencies for brain syndromes. There is no evidence for assuming that the real incidence increased in the 1970's and 1980's whereas, in contrast, medical treatment facilities for infections or traumas of the brain have improved considerably. It is more likely that the prevailing popularity of the minimal brain dysfunction (MBD) concept in the local school of thought, as in many other countries, was mainly responsible for this increase in frequencies until it came under attack in the 1980 's $(11,17)$ and was gradually discarded. In addition, the introduction of a separate axis for specific developmental disorders in the multiaxial scheme of classification contributed to the decline in frequencies of brain syndromes.
The considerable decline of the distribution of mental retardation in the same period of the 1970's and 1980 's can certainly be attributed to the fact that child psychiatry lost more and more of this clientele to other disciplines. Both paediatrics, with its increasing diagnostic facilities, and special education, with its increasing responsibilities in the area of training and supervision largely contributed to this trend, which became apparent not only in Switzerland but also in other developed countries. This trend is also quite obvious in the research literature in which child psychiatry, in contrast to the basic biological sciences and psychology, has contributed little in the recent past $(5,6,8)$.

Finally, in contrast to the waning responsibility of child psychiatry for the assessment and treatment of mentally retarded persons, the present study indicates that there has been a strong trend in the recent past for children with developmental disorders to be diagnosed more frequently. Closer inspection of the data revealed that this effect coincided with the introduction of the ICD-9 and the multiaxial system of classification to the service. With its emphasis on developmental disorders on a separate axis and, thus, the provision for considering multiple diagnoses, this change in the diagnostic system was mainly responsible for changes in prevalence rates that certainly do not represent true increases in morbidity. In contrast, it has to be assumed that with a one-dimensional scheme for diagnostic assessment, a large number of developmental delays associated with a prevailing psychiatric syndrome were not considered in the statistics.

In conclusion, this analysis of trends in child and adolescent psychiatric disorders over seventy years in the most densely populated canton of Switzerland shows a steady but not very pronounced increase of administrative prevalence rates over time. Although comparative figures on the true prevalence rates are lacking, the conclusion is justified that these figures based on attendance rates represent only a minor proportion of the actual morbidity. The sex distribution with a strong and continuing male preponderance and the age distribution of the present sample is in accordance with current knowledge. The changes in the use of diagnoses over time reflects to a greater extent the changes of nosology in child and adolescent psychiatry rather than any hypothetical changes in true morbidity.

Acknowledgements The author is indebted to his predecessors Professor Dr. J. Lutz and the late Professor Dr. R. J. Corboz, who laid the groundwork for this study and to R. Kannenberg and S. Willener for their assistance with data computation. 


\section{References}

1. American Psychiatric Association (1980) Diagnostic and Statistical Manual of Mental Disorders (DSM-III) (Third Edition ed). APA, Washington D.C.

2. American Psychiatric Association (1987) Diagnostic and Statistical Manual of Mental Disorders (DSM-III-R) (Third Edition ed). APA, Washington D.C.

3. American Psychiatric Association (1994) Diagnostic and Statistical Manual of Mental Disorders (DSM-IV) (4th ed). APA, Washington, D.C.

4. Brandenburg $\mathrm{N} A$, Friedman $\mathrm{R} \mathrm{M}$, Friedman, RM, Silver SE (1990) The epidemiology of childhood psychiatric disorders: prevalence findings from recent studies. Journal of the American Academy of Child and Adolescent Psychiatry 29:76-83

5. Bregman ID (1991) Current developments in the understanding of mental retardation. Part II: Psychopathology. Journal of the American Academy of Child and Adolescent Psychiatry 30:861-872

6. Bregman JD, Hodapp RM (1991) Current developments in the understanding of mental retardation. Part I: Biological and phenomenological perspectives. Journal of the American Academy of Child and Adolescent Psychiatry 5:707719

7. Costello EJ, Burns BJ, Angold A, Leaf PJ (1993) How can epidemiology improve mental health services for children and adolescents? Journal of the American Academy of Child and Adolescent Psychiatry 32:1106-1113

8. Fraser W I, Rao JM (1991) Recent studies of mentally handicapped young people's behaviour. Journal of Child Psychology and Psychiatry and Allied Disciplines 32:79-108
9. Gould MS, Wunsch-Hitzig R, Dohrenwend B (1981) Estimating the prevalence of childhood psychopathology - a critical review. Journal of the American Academy of Child and Adolescent Psychiatry $20: 462-476$

10. Hill J, Rutter M (1994) Personality Disorders. In: Rutter M, Taylor E, Hersov L (eds) (3rd ed). Blackwell, Oxford

11. Kalverboer AF (1993) Neurobehavioural relationships in children - New facts, new fictions. Early Human Development 34:169-177

12. Larsen F W, Dahl, V, Hallum E (1990) A 30-year follow-up study of a child psychiatric clientel. I: Demographic description. Acta Psychiatrica Scandinavica 81:39-45

13. Links PS (1983) Community surveys of the prevalence of childhood psychiatric disorder: a review. Child Development 54:531-548

14. Offord DR (1985) Child psychiatric disorders - prevalence and perspectives. Psychiatric Clinics of North America 8.

15. Offord DR, Boyle MH, Fleming JE, Blum HM, Rac-Grang N (1989) Summary of selected results. Canadian Journal of Psychiatry 34:483-491

16. Östman O (1991) Child and adolescent psychiatric patients in adulthood. Acta Psychiatrica Scandinavica 84:40-45

17. Rutter M (1982) Syndromes attributed to "minimal brain dysfunction" in childhood. American Journal of Psychiatry 139:21-33

18. Rutter M (1989) Isle of Wight Revisited: Twenty-five years of child psychiatric epidemiology. Journal of the American Academy of Child and Adolescent Psychiatry 28:633-653
19. Rutter M, Tizard J, Whitmore K (1970) Education, Health, and Behaviour. Longman, London

20. Rutter M, Shaffer D, Sturge C (1975) University of London, London

21. Steinhausen H-C (1992) Sex differences in developmental psychopathology. In: Remschmidt H, Schmidt $\mathrm{MH}$ (eds) Hogrefe \& Huber Publishers, Lewiston, New York; Toronto; Bern; Göttingen

22. Steinhausen H-C, Erdin A (1991a) A comparison of ICD-9 and ICD-10 diagnoses of child and adolescent psychiatric disorder. Journal of Child Psychology and Psychiatry 32:909-920

23. Steinhausen H-C, Erdin A (1991b) The interrater reliability of child and adolescent psychiatric disorder in ICD-10. Journal of Child Psychology and Psychiatry 32:921-928

24. Steinhausen H-C, Erdin A (1992a) Abnormal psychosocial situations and ICD10 diagnoses in children and adolescents attending a psychiatric service. Journal of Child Psychology and Psychiatry 33:731-740

25. Steinhausen H-C, Erdin A (1992b) Correlates of the DSM-III-R global assessment of functioning scale in patients attending a child and adolescent psychiatric service. European Child and Adolescent Psychiatry 1:105-110

26. World Health Organization (1978) International Classification of Diseases. Ninth Revision. Author, Geneva

27. World Health Organization (1992) The ICD-10 Classification of Mental and Behavioural Disorders. Clinical descriptions and guidelines. Author, Geneva 